\title{
СОЦИОЛОГИЯ
}

И СОЦИАЛЬНЫЕ ТЕХНОЛОГИИ

DOI: https://doi.org/10.15688/lp.jvolsu.2020.3.3

UDC 316.334

LBC 60.561 .2

\section{DIGITAL INEQUALITY: INTERNET TECHNOLOGIES IN ACTIVATION OF CONSUMER BEHAVIOR ${ }^{1}$}

\author{
Nina N. Ivashinenko \\ Lobachevsky State University, Nizhny Novgorod, Russian Federation; \\ University of Glasgow, Glasgow, United Kingdom \\ Mikhail L. Teodorovich \\ Lobachevsky State University, Nizhny Novgorod, Russian Federation \\ Alla A. Varyzgina \\ Lobachevsky State University, Nizhny Novgorod, Russian Federation
}

\begin{abstract}
The paper is focused on the analysis of the territorial distribution of digital inequality in the Russian region and its influence on the level of online trading in the region. The paper presents the results of monitoring studies of the development of competition and of anti-monopoly regulation in the Nizhny Novgorod region, conducted since 2014, and the results of the last survey in 2018 (comparable methodology was used) on 3,000 respondents out of 20 districts of the region, different by their socio-economic situation. The sample is \&ิ territorially random, representing the population by sex and age. The authors use the concept of digital inequality $\checkmark$ of the third generation developed by Ragnedda (Ragnedda, 2017) for study of digital inequality in shopping online possibilities. This approach defines digital inequality not only as an information and technological and educational elements in differences of social groups and communities in the Internet access, but also as an inequality of the I advantages that groups might get from its use. This type of inequality cannot be overcome only due to technological and popularizing decisions; inequality should be controlled by the state and communities to prevent its escalation 诺 and of excluding entire territories from social and economic development. The paper presents the invented methodology for the typology of administrative municipalities of the region, which helps in implementation of state control over competition. All territories of the region were divided into four zones with different degree of government $\sum_{5}$ regulation needed. It helps to reduce the imbalance in market development and to lower the digital divide in Internet 商 shopping. A study of the interrelation between online shopping and retail population satisfaction in administrative r. regions of Russian regions shows the urgent need to expand municipal competition development maps to include Shopping online indicators.
\end{abstract}

Key words: digital divide, online shopping, regional development, consumer behaviour, quality of life. 
УДК 316.334

ББК 60.561 .2

\title{
ЦИФРОВОЕ НЕРАВЕНСТВО: ИНТЕРНЕТ-ТЕХНОЛОГИИ В АКТИВИЗАЦИИ ПОТРЕБИТЕЛЬСКОГО ПОВЕДЕНИЯ ${ }^{1}$
}

\author{
Нина Николаевна Ивашиненко \\ Нижегородский государственный университет им. Н.И. Лобачевского, г. Нижний Новгород, \\ Российская Федерация; Университет Глазго, г. Глазго, Великобритания
}

\section{Михаил Леонидович Теодорович}

Нижегородский государственный университет им. Н.И. Лобачевского, г. Нижний Новгород, Российская Федерация

\section{Алла Александровна Варызгина}

Нижегородский государственный университет им. Н.И. Лобачевского, г. Нижний Новгород, Российская Федерация

\begin{abstract}
Аннотация. Данная статья посвящена анализу территориального распределения цифрового неравенства в масштабах российского региона и его проявления на уровне интернет-торговли. Эмпирическая часть статьи базируется на результатах мониторинговых исследований развития конкуренции и антимонопольного регулирования в Нижегородской области, проводимого по сопоставимой методике с 2014 г., и результатах последнего опроса 2018 года. В ходе исследования приняли участие 3000 респондентов в 20 районах региона, репрезентирующих область по социально-экономическому положению. Выборка территориальная случайна, репрезентирующая население по поло-возрастному составу. Исследование процессов цифрового неравенства и его проявления в процессе использования возможностей интернет-торговлии изучалось на базе концепции цифрового неравенства третьего поколения, разработанного Рагнеддой (Ragnedda, 2017). Данный подход определяет цифровое неравенство не просто как информационно-технологичное и образовательное различие социальных групп и территорий в доступе к интернет, сколько как неравенство преимуществ, извлекаемых группами из его использования. Этот тип неравенств не может быть преодолен только за счет технологических и популяризаторских решений, он нуждается в постоянном контроле со стороны государства и сообществ с целью недопущения его эскалации и возникновения ситуации исключения целых территорий из основных процессов развития. На базе анализа разработана методика типологизации административных муниципальных образований региона для реализации мер государственного контроля над процессами конкуренции. Все территории региона могут быть разделены на четыре зоны в разной степени нуждающиеся в государственном регулировании, позволяющем сгладить дисбаланс рыночного развития и снизить остроту проявления цифрового неравенства в интернет-торговле. Изучение взаимосвязи между интернет-торговлей и удовлетворенностью населения розничной торговлей в различных административных районах российских регионов показывает насущную необходимость расширения муниципальных дорожных карт развития конкуренции в плане включения в них индикаторов интернет-торговли.

Ключевые слова: цифровое неравенство, интернет-торговля, региональное развитие, поведение потребителей, качество жизни населения.
\end{abstract}

Начало проблемы цифрового неравенства (digital divide) относят к концу двадцатого века и первым исследованиям, связанным с распространением интернет и сетевых сообществ [Castels 1996; Dijk 1999]. Поставленная как научная проблема в ситуации, когда только 3 \% населения имели доступ к Интернет, проблема цифрового неравенства прошла значительный путь от эйфории по поводу демократизации общества за счет новых тех- нологий [Compaine 2001], до пессимистических прогнозов усугубления неравенства [Norris 2001]. Несмотря на сравнительно короткий исторический период в развитии проблемы цифрового неравенства можно выделить три основных этапа [Ragnedda 2017].

Первый этап определения цифрового неравенства и его изучения связан с дихотомическими концепциями, выстраиваемыми вокруг социальных групп, имеющих доступ в 
Интернет, и не имеющими такого доступа [Correa 2010]. Инициаторами этого направления исследований в разных странах выступали организации, вовлеченные в процессы предоставления телекоммуникаций или их регулирования (NTIA). Особенно активно на данном этапе работы ученых пересекались с высказываниями политиков разных стран, заявлявших о важности обеспечения равенства населения в доступе к Интернет [DiMaggio et al. 2004; Hargittai, Walejko 2008; Шарифьянов, Гайнанов 2016]. Технологически-инновационный подход первого уровня к определению цифрового неравенства развивался в направлении изучения возможности преодоления диспаритета доступа к интернету, как в глобальном разрезе - между странами, так и внутри стран в рамках различных территорий. Географический подход первого этапа развития концепции цифрового неравенства также опирался на концепции распространения инноваций в обществе в целом [Thierer 2000] и скорости их освоения. Технологически-инновационный подход первого уровня к определению цифрового неравенства остается базовым для дальнейшего развития понимания процессов социального неравенства и роли цифровых технологий в нем, но далеко не исчерпывает весь спектр проблем доступа (access) к информационным ресурсам.

Второй этап развития концепции цифрового неравенства связан с переходом от дихотомической концепции доступа, разделяющей общество на имеющих возможность пользоваться цифровыми технологиями и не имеющих, к многомерной концепции доступа, анализирующей, как используется интернет. Наряду с понятием доступа вводится понятие цифровых навыков (digital skills) и цифрового капитала (digital capital) [Ragnedda 2017]. Цифровое неравенство взаимоувязывается с неравенством в получении знаний и навыков, которое в офлайн сообществе имеет доказанную связь с социальными стратами [Волченко 2016; Трошина, Рощина 2013]. Более молодые и состоятельно-обеспеченные группы имеют в среднем более высокий уровень знаний, необходимый для работы с Интернет, чем менее обеспеченные и более возрастные группы. По мере ускорения смены технологических инноваций этот разрыв в знаниях имеет тенденцию нарастать [Norris 2001]. Технологическая проблема, такая как рост скорости предоставления и объема данных доступа в Интернет, дополняется проблемой различия в знаниях и мотивации в их получении. Мотивационная составляющая приобретает важнейшее значение, усиливая важность социально-экономических подходов к решению проблем цифрового неравенства. Концепция (MAD'swall - Motivation, Accessand Digital capital) анализирует как три основные составляющие - мотивация, доступ и цифровой капитал - влияют на цифровое неравенство, формируя и воспроизводя его на базе существующей в обществе социальной стратификации.

Третьим этапом в развитии концепции цифрового неравенства стал логичный переход от вопросов: «Кто имеет технический доступ?» и «Какие знания используются?» к вопросу «Какая прибыль извлекается из использования интернет технологий?» Многочисленные исследования показывают, что знания и цифровой капитал не конвертируются автоматически в финансовый и социальный капитал. Как показывают исследования Ragnedda и Muscher [Ragnedda, Muschert 2016], социально-экономический статус пользователя влияет на способ получения информации и знаний, и увеличивает разрыв между бедными и богатыми. Цифровое неравенство имеет тенденцию не только базироваться на уже существующем социальном неравенстве, но и усиливать его [Волченко 2016]. В этом случае программы преодоления социального неравенства и борьбы с бедностью должны перестаиваться и учитывать новые инфраструктурные факторы.

Для России проблема цифрового неравенства получила свою актуальность также сравнительно недавно. Около трети территорий российских регионов не имеют полного доступа в Интернет, и для них актуальна проблематика первого этапа цифрового неравенства, обусловленная технологическими проблемами. В тоже время виртуальное российское сообщество растет стремительными темпами, порождая соседство в рамках одного региона как проблем технологического доступа, так и проблем навыков и знаний. Наряду с территориями со слабой технологической инфраструктурой в рамках одного и того же ре- 
гиона появляются территории с высокоразвитыми кластерами пользователей, покупающих в интернете определенный вид товаров и услуг, которые начинают вытеснять точки реальной торговли. Так, например, происходит в секторах продажи авиа- и железнодорожных билетов, количество точек реальных продаж которых сократилось за последние годы. В результате потенциальные покупатели, не имеющие возможности покупать билеты онлайн, оказались в невыгодной позиции в плане реализации потребительного поведения. Учитывая тенденцию рынка быстро разворачиваться в ниши, интересные, в первую очередь, высоко ресурсным покупателям, основная масса которых комфортно себя чувствует в интернет, возникает вопрос: как будет выстраиваться баланс между интернет- и реальной торговлей на определенных территориях? Как государство как регулятор процессов конкуренции, с одной стороны, и процессов контроля социального неравенства, с другой стороны, может учитывать данную ситуацию? Опираясь на подход Массимо Рагнедды [Castells 1996] к определению цифрового неравенства как различия в получении выгод от использования интернет, мы проанализировали ситуацию на примере одного из российских регионов - Нижегородской области в части одного из пластов использования Интернета - интернет-торговли.

\section{Описание методологии исследования}

Наш исследовательский коллектив более 10 лет занимается вопросами регионального развития и конкуренции как одного из двигателей экономических процессов. В 2014 г. кафедра экономической социологии факультета социальных наук ННГУ им. Н.И. Лобачевского и Научно-исследовательский центр ЭОН по инициативе и поддержке Нижегородского территориального управления Федеральной антимонопольной службы Российской Федерации провели исследование, посвященное развитию конкуренции и совершенствованию антимонопольной политики в Нижегородской области. В ходе проекта была разработана методика оценки развития конкуренции как элемента механизма повышения качества жизни населения малого города с применени- ем сравнительного анализа состояния рынков потребительских товаров и услуг столицы региона и районных центров. Методика разрабатывалась в соответствие с дорожной картой развития конкуренции и совершенствования антимонопольной политики [План... web], реализуемой на уровне органов местного самоуправления, и позволила измерять уровень эффективности ее исполнения. Данная методика получила высокую оценку и была рекомендована ФАС для использования в других регионах страны. Мониторинговые исследования проводились ежегодно. В 2018 г. мониторинг удовлетворенности потребителей качеством товаров и услуг на товарных рынках и состоянием ценовой конкуренции в Нижегородской области проводился кафедрой социологии проектной деятельности и проконкурентного регулирования ННГУ им. Н.И. Лобачевского совместно с центром исследования социальных систем ННГУ им. Н.И. Лобачевского и НИЦ ЭОН в рамках договора о сотрудничестве между Нижегородским государственным университетом им. Н.И. Лобачевского и Министерством экономического развития и конкуренции Нижегородской области. В ходе исследования были опрошены 3000 респондентов, проживающих в 20 районах области, включая городские округа и Нижний Новгород. Выборка - районированная, репрезентирующая районы Нижегородской области по численности населения и социально-экономическому развитию. Внутри района случайная маршрутная выборка контролировалась по поло-возрастному составу и репрезентировала жителей района.

Нижегородская область по уровню социально-экономического развития, трудовому потенциалу и техническому доступу населения к Интернет относится к средне статистическим российским регионам. По данным Нижегородстата, доля домашних хозяйств, имеющих в целом по области доступ в интернет составляла в 2015 году 73,5 \%, в 2016 году 79,8 \% и достигла в 2017-80,5 \% [Территориальный орган... web]. При этом различие между сельскими территориями в целом по области между городской и сельскими местностями сокращается и составило в 2017 г. 4,8 \%, вместо 8,7 \% в 2016 году. Тем не менее общая статистика не отражает специфики развития конкретных территорий. 
Для анализа географической составляющей Нижегородской области, необходимой для совершенствования государственной политики регулирования конкуренции, использовалось два типа классификации: активность использования Интернет-торговли и удовлетворенность населения состоянием розничной торговли на данной территории. В результате применения этих двух осей все районы Нижегородской области могут быть разделены на четыре квадранта:

1. Территории с повышенным уровнем использования интернет-торговли и развитой инфраструктурой розничной торговли (50\%).

2. Территории с развитой розничной торговлей и менее развитой интернет-торговлей $(24 \%)$.

3. Территории с менее развитой розничной торговлей, но с более высоким распространением интернет-торговли (13\%).

4. Территории с менее развитой и розничной, и интернет-торговлей (13\%).

\section{Зона благополучия: развитая розничная и онлайн торговля}

В зону относительного благополучия входят районы столицы региона - Нижнего Новгорода и прилегающие к ней города, образующие по сути мегаполис. Учитывая размеры населения Нижнего Новгорода по отношению к остальным территориям области в целом, не удивительно, что данная зона достаточно большая и составляет около половины жителей области.

Сосредотачиваемые здесь ресурсы спроса со стороны населения позволяют обоим рынкам офлайн и онлайн торговли выходить на приемлемые показатели, создавая высокий уровень комфортности жизни населения по сравнению с другими территориями. В зону благополучия входят наиболее развитые в информационно-технологическом плане территории. Жители в большинстве своем имеют свободный доступ к цифровым технологиям и являются активными интернет-пользователями. Обладая более высоким социальным капиталом, в частности, имея более высокий уровень образования (34\% жителей этой территории имеют высшее образование) и материального благосостояния, жители имеют возможность более эффективно использовать имеющиеся у них цифровые возможности, чем жители других территориальных зон.

На рассматриваемых территориях хорошо развита сеть розничной торговли, жители в большей степени, чем в других географических зонах, удовлетворены возможностью выбора (85\%) и качеством (81\%) товаров, реализуемых через систему розничной торговли, а также испытывают меньшую неудовлетворенность существующим уровнем цен.

Степень развитости интернет-инфраструктуры по оценкам населения вполне достаточна, в целом вызывает удовлетворение уровень цен на услуги и их качество, возможность выбора поставщиков услуг доступа в интернет. Почти половина населения зоны (41\%) активно пользуется онлайн торговлей, причем четверть из них совершает покупки в интернете как минимум 1 раз в месяц, а половина интернет-покупателей совершает покупки не чаще раз в 2-3 месяца. Основные причины, сдерживающие их активность в пространстве интернет-торговли, связаны, прежде всего, с высокой степенью развитости розничной торговли по месту жительства (нужные товары проще купить в магазине) и определенным нежеланием в этих условиях идти на риск, присутствующий при покупке товаров в интернете и связанный, прежде всего, с вероятностью необходимости возврата неподходящего товара. Наиболее привлекательными чертами онлайн торговли для населения является гибкость в системе оплаты и соотношение цены и качества товаров. Вызывает удовлетворение доступность пунктов получения товаров.

В зоне наиболее развитой офлайн и онлайн торговли необходимо минимальное государственное регулирование на уровне текущего контроля за функционированием торговых площадок.

\section{Зона развитой розничной торговли \\ и менее развитой интернет-торговли}

В зоне с преимущественным развитием реальной торговлей и пониженной активностью онлайн торговли оказываются экономически развитые районы области, при этом рас- 
стояние до областного центра не имеет принципиального значения для исследуемых процессов: сюда в равной степени входят и средне удаленные, и далеко расположенные от областного центра районы.

Население, проживающее в районах в пределах выделенной географической зоны, удовлетворено текущим состоянием розничной торговли по месту проживания, прежде всего, качеством реализуемых товаров (81\%). При этом по сравнению с областным центром, несколько снижена удовлетворенность существующей возможностью выбора товаров и уровнем цен.

В выделенных районах по оценкам населения меньше развита инфраструктура, связанная с предоставлением населению доступа в интернет: недостаточен выбор услуг, связанных с обеспечением доступа к интернету и уровень цен на них; качество связи в целом признается удовлетворительным. Наданных территориях прослеживается более выраженный запрос на дальнейшее развитие этих услуг. Более низкий уровень развития интернет-инфраструктуры в этих городах и районах ограничивает доступ населения к услугам онлайн-торговли. Потребители данных территорий имеют более скромное материальное положение, чем в областном центре и мегаполисе, а также более низкий уровень образования и квалификации, что в совокупности с менее развитыми техническими возможностями цифровой среды становится фактором, ограничивающим их потребительскую активность в цифровом торговом пространстве.

На данный момент только пятая часть населения совершала покупки в интернет (22 \%), как правило, это не частые покупатели: покупки совершаются лишь эпизодически, чаще раз в полгода. Также для пятой части населения причиной неучастия в онлайн торговле является отсутствие доступа в интернет (22 \%). Возможность совершать покупки онлайн привлекательна для населения этих территорий, их в меньшей степени пугает вероятность необходимости возврата неподходящего товара, но большей сложностью является более высокая стоимость доставки товара по сравнению с ситуацией в областном центре.
На данных территориях есть большой потенциал развития онлайн торговли и увеличения ее объемов, который на данный момент сдерживается сохраняющимся цифровым и инфраструктурным неравенством. Необходимо государственное регулирование, направленное на снижение уровня цифрового неравенства на территориях, на информационно-технологическое развитие территорий.

\section{Зона с более высоким}

\section{распространением интернет-торговли}

В зону с более высоким распространением интернет-торговли преимущественно входят удаленные от областного центра районы с более сложным экономическим положением.

Население, проживающее на этих территориях, дает существенно более низкие оценки уровню развития розничной торговли. При этом вызывает особое недовольство существующий уровень цен, недостаточные возможности выбора товара.

Инфраструктура доступа в интернет на территориях развита в достаточной степени, но четверть населения территорий выражает недовольство, связанное в основном с недостаточным выбором поставщиков услуг доступа в интернет, тогда как качество имеющихся услуг оценивается достаточно высоко. Существующий на данный момент уровень развития розничной торговли на территории не удовлетворяет в должной мере потребности населения, что в сочетании с удовлетворительным уровнем развития услуг интернет-связи на территориях позволяет значительному числу граждан (33 \%) реализовывать покупательские потребности с помощью онлайн торговли, при этом значительная часть из них являются активными покупателями, регулярно совершающими покупки онлайн. На фоне заинтересованности населения в развитии онлайн торговли сдерживающими факторами выступают инфраструктурные ограничения, связанные с недостаточным числом пунктов выдачи заказов, а также стоимостью доставки. Одним из факторов, повышающих привлекательность онлайн-торговли по сравнению с розничной торговлей, является более низкая цена товаров. Значимость этого фактора по- 
вышена в ситуации более низкого уровня материального благосостояния населения этих территорий.

Более развитая онлайн торговля на данных территориях отчасти компенсирует недостаточный уровень развития офлайн торговли. Востребована деятельность государственных структур, направленная на развитие технической составляющей информационной среды территорий как необходимого условия для активизации потребительского поведения в цифровом торговом пространстве.

\section{Зона наименее развитой торговли}

В зону наибольшего неблагополучия входят районы, отдаленные от областного центра и не имеющие достаточно развитой инфраструктуры, необходимой для развития и традиционной розничной, и онлайн-торговли. Социально-экономическая ситуация в этих районах наиболее сложная. Проживающее в этих районах население низкоресурсное: имеет наиболее низкий уровень образования ( $37 \%$ не имеет ни средне специального, ни тем более высшего образования), а также наиболее низкий уровень материального благосостояния (41\% оценивает свое положение как проживание в условиях бедности), как следствие, выше доля людей, вынужденных работать за пределами собственного населенного пункта (23\%), повышена доля безработных (8\%). В районах проживает более возрастное население (доля пенсионеров - $33 \%$ ).

Как в плане состояния розничной торговли, так и развития онлайн-торговли ситуация на этих территориях также наиболее сложная. Жители этих территорий наиболее часто выражают неудовлетворенность состоянием розничной торговли, особенно уровнем цен (72 \%). Этот критерий для них имеет наиболее важное значение в силу наиболее низкого уровня материального благосостояния (значительная часть жителей проживает на грани или в состоянии бедности).

Доступ в интернет на этих территориях также сильно ограничен, больше половины населения отмечает недостаточное развитие этих услуг (56\%). Особое недовольство связано с высокой стоимостью услуг (43\%) и недостаточным выбором товаров (51\%).
В существующей ситуации цифрового неравенства жители выражают наиболее яркий запрос на развитие торговли. В представлении жителей, для этих территорий более важно развитие интернет-торговли, способной скомпенсировать недостаточное развитие розничной торговли на территориях.

В силу неразвитости инфраструктуры, необходимой для развития онлайн торговли, только незначительная часть населения (14\%) имеет опыт (как правило, единичный) совершения покупок в интернет. Как главное препятствие жители упоминают долгий срок и неразвитость структуры доставки. Наиболее низкий уровень материального благосостояния населения также является фактором, повышающим интерес жителей к возможности совершать покупки онлайн: большее, чем для других, значение имеет возможность найти более дешевый товар приемлемого качества.

Районы, входящие в зону неблагополучия, требуют наибольшего внимания со стороны государства в направлении контроля за развитием ситуации. Районы не имеют достаточного количества собственных ресурсов для развития рынка онлайн и традиционной розничной торговли. На данных территориях в большей степени проявляется цифровое неравенство, являющееся одним из сдерживающих факторов социально-экономического развития территорий.

$$
* * *
$$

Понимание цифрового неравенства как социальной проблемы, порождаемой целым комплексом факторов, не только и не столько технологическим доступом, сколько различием в цифровых капиталах и стратегиях использования новых технологий меняет повестку и фокус государственных программ регулирования этих процессов. Внедрение новых информационных технологий становится важнейшим фактором развития территорий. Территории, на которых отсутствует развитая инфраструктура, начинают не просто исключаться из общего процесса, но стремительно терять в качестве жизни населения. Население, имеющее более высокие капиталы знаний и умений в интернет-сферах, стремится покинуть территории с низко развитой инфраструк- 
турой. В результате из данных районов уезжает наиболее обеспеченная часть потребителей, что сказывается и на реальной торговле (снижение платежеспособного спроса). На этих территориях проявляются все три вида цифрового неравенства: технологического доступа, знаний и умений и желания использовать их для получения выгоды от интернетторговли.

Территории, где развитие интернет-торговли компенсирует неразвитость конкуренции в сфере реальной торговли, выглядят несколько оптимистичнее с точки зрения технического прогресса. В то же время в них создает напряженность внутри района между социальными группами, которые могут и хотят пользоваться интернет-торговлей, и группами, которые не хотят пользоваться интернет-торговлей, но реальной пользоваться не могут. Реальная торговля в этих районах начинает терять в своем качестве вследствие снижения спроса со стороны уходящих в виртуальное шопинг-пространство пользователей. Если в полностью неблагополучных районах наблюдается реальная мобильность и отток населения, но в этом типе районов обе группы пользователей проживают вместе, но поведение одних в интернет торговле начинает сужать возможности выбора других в сфере реальной торговли.

Таким образом, одновременное функционирование на территории нескольких форм электронного и неэлектронного потребительского поведения ставит сложные задачи регулирования процессов конкуренции с целью повышения качества жизни населения. Одним из вариантов развития процессов государственного регулирования видится разработка новых индикаторов в дорожные карты развития конкуренции для регионов и муниципалитетов, где наряду с удовлетворенностью населением состояния конкуренции на различных рынках предлагается проводить мониторинги развитости электронной торговли и баланса между различными формами торговли. Подход к цифровому неравенству как чисто технической проблеме может усугублять формирующееся неравенство и усиливать давление на рынки реальной торговли, особенно для слабо защищенных слоев населения.

\section{ПРИМЕЧАНИЕ}

\begin{abstract}
${ }^{1}$ Исследование проведено в рамках договоpa о сотрудничестве между Нижегородским государственным университетом им. Н.И. Лобачевского и Министерством экономического развития и конкуренции Нижегородской области.

The study was conducted under a cooperation agreement between the Lobachevsky State University of Nizhny Novgorod and the Ministry of economic development and competition of the Nizhny Novgorod region.
\end{abstract}

\section{СПИСОК ЛИТЕРАТУРЫ}

Волченко 2016 - Волченко О.В. Динамика цифрового неравенства в России // Мониторинг общественного мнения: Экономические и социальные перемены. 2016. № 5. С. 163-182.

План... web - План мероприятий («дорожная карта») по содействию развития конкуренции в Нижегородской области : утв. распоряжением губернатора Нижегородской области 30 декабря 2014 г. № 2268-p // https://minec. government-nnov.ru/?id=168961.

Территориальный орган... web - Территориальный орган Федеральной службы государственной статистики по Нижегородской области. Официальная статистика. Уровень жизни // http:// nizhstat.old.gks.ru/wps/wcm/connect/rosstat_ts/ nizhstat/ru/statistics/standards_of_life/

Трошина, Рощина 2013 - Трошина А. $\bar{A} ., \bar{P}$ Рощина Я.М. Цифровое неравенство россиян в сфере медиапотребления // Козырев П.М. (ред.). Вестник Российского мониторинга экономического положения и здоровья населения НИУ ВШЭ (RLMS - HSE). Вып. 3. М.: НИУ ВШЭ, 2013. С. 187-203.

Шарифьянов, Гайнанов 2016 - Шарифьянов Т.Ф., Гайнанов Д.А. Модели преодоления цифрового неравенства в малых удаленных населенных пунктах на основе государственно-частного партнерства // Региональная экономика: теория и практика. 2016. № 8 (431). С. 19-32.

Castells 1996 - Castells M. The Information Age: Economy, Society and Culture. Vol. I: The Rise of the Network Society. Oxford: Blackwell, 1996.

Compaine 2001 - Compaine B.M. The Digital: Facing a Crisis or Creating a Myth? Boston, MA: MIT Press, 2001.

Correa 2010 - Correa T. The participation divide among 'online experts': Experience, skills and psychological factors as predictors of college students' web content creation // Journal of Computer-Mediated Communication. 2010. № 16 (1). P. 71-92. 
DiMaggio et al. 2004 - DiMaggio P., Hargittai E., Celeste $C$., Shafer $S$. Digital Inequality: From Unequal Access to Differentiated Use // Neckerman K. (ed.). Social Inequality. New York: Russell Sage Foundation, 2004. P. 355-400.

Dijk 1999 -Dijk van J.A.G.M. The Network Society: Social Aspects of the New Media. London, Thousand Oaks, CA, and New Delhi: Sage Publications, 1999.

Hargittai, Walejko 2008 - Hargittai E., Walejko G. The participation divide: Content creation and sharing in the digital age // Information, Communication \& Society. 2008. № 11 (2). P. 239-256.

Norris 2001 - Norris P. Digital Divide: Civic Engagement, Information Poverty and the Internet in Democratic Societies. New York: Cambridge University Press, 2001.

Ragnedda 2017 - Ragnedda M. The third digital divide. A Weberian Approach to Digital Inequalities. New York, NY: Routledge, 2017.

Ragnedda, Muschert 2016-Ragnedda M., Muschert G.W. Theorizing Digital Divides and Digital Inequalities // Servaes J., Oyedemi T. (eds.). Social Inequalities, Media and Communication: A Global Perspective. London: Lexington Books, 2016. P. 23-35.

Thierer 2000 - Thierer $A$. Is the digital divide a virtual reality? // Consumers' Research Magazine. 2000. № 83 (7). P. 16-21.

\section{REFERENCES}

Volchenko O.V., 2016. Dynamics of digital inequality in Russia. Monitoring of Public Opinion: Economic and Social Changes, no. 5, pp. 163-182.

The "road map" to promote the development of competition in the Nizhny Novgorod region, 2014. Approved by order of the Governor of the Nizhny Novgorod Region on December 30, No. 2268-p. URL: https://minec.governmentnnov.ru/?id=168961.

The official website of the Territorial Authority of the Federal State Statistics Service for the Nizhny Novgorod Region. URL: http://nizhstat.old. gks.ru/wps/wcm/connect/rosstat_ts/nizhstat/ ru/statistics/standards_of_life.
Troshina A.A., Roshchina Ya.M., 2013. Digital inequality of Russians in the field of media consumption. Kozyreva P.M. (ed). Bulletin of the Russian Monitoring of the Economic Situation and Public Health of the Higher School of Economics (RLMS - HSE), iss. 3. Moscow, HSE, pp. 187-203.

Sharifyanov T.F., Gaynanov D.A., 2016. Models for overcoming the digital divide in small remote settlements based on public-private partnerships. Regional Economics: Theory and Practice, no. 8 (431), pp. 19-32.

Castells M., 1996. The Information Age: Economy, Society and Culture. Vol. I: The Rise of the Network Society. Oxford, Blackwell.

Compaine B.M., 2001. The Digital: Facing a Crisis or Creating a Myth? Boston, MA, MIT Press.

Correa T., 2010. The participation divide among 'online experts': Experience, skills and psychological factors as predictors of college students' web content creation. Journal of ComputerMediated Communication, no 16(1), pp. 71-92.

DiMaggio P., Hargittai E., CelesteC., Shafer S., 2004. Digital Inequality: From Unequal Access to Differentiated Use. Neckerman K. (ed.). Social Inequality. New York, Russell Sage Foundation, pp. 355-400.

Dijk Van J.A.G.M., 1999. The Network Society: Social Aspects of the New Media. London, Thousand Oaks, CA, and New Delhi, Sage Publications.

Hargittai E., Walejko G., 2008. The participation divide: Content creation and sharing in the digital age. Information, Communication \& Society, no. 11 (2), pp. 239-256.

Norris P., 2001. Digital Divide: Civic Engagement, Information Poverty and the Internet in Democratic Societies. New York, Cambridge University Press.

Ragnedda M., 2017. The third digital divide. A Weberian Approach to Digital Inequalities. NewYork, NY, Routledge.

Ragnedda M., Muschert G.W., 2016. Theorizing Digital Divides and Digital Inequalities. Jan Servaes and Toks Oyedemi (eds.). Social Inequalities, Media and Communication: A Global Perspective. London, Lexington Books, pp. 23-35.

Thierer A., 2000. Is the digital divide a virtual reality? Consumers' Research Magazine, no. 83 (7), pp. 16-21. 


\section{Information About the Authors}

Nina N. Ivashinenko, Doctor of Sciences (Economics), Professor, Head of the Research Center for Social Systems, Lobachevsky State University of Nizhny Novgorod, Universitetskiy Lane, 7-209, 603000 Nizhny Novgorod, Russian Federation; Honorary Researcher, School of Social and Political Sciences, University of Glasgow, University Avenue, G12 8QQ Glasgow, United Kingdom, nni@fsn.unn.ru, nina.ivashinenko@glasgow.ac.uk, https://orcid.org/0000-0001-5242-169X

Mikhail L. Teodorovich, Doctor of Sciences (Sociology), Head of the Department of Project Agency and Pro-Competitive Policy, Lobachevsky State University of Nizhny Novgorod, Universitetskiy Lane, 7-209, 603000 Nizhny Novgorod, Russian Federation, mt231@fsn.unn.ru, https://orcid.org/0000-0002-2136-9101

Alla A. Varyzgina, Candidate of Sciences (Sociology), Senior Lecturer, Department of Project Agency and Pro-Competitive Policy, Lobachevsky State University of Nizhny Novgorod, Universitetskiy Lane, 7-209, 603000 Nizhny Novgorod, Russian Federation, alvar@fsn.unn.ru, https://orcid.org/0000-0002-1406-836X

\section{Информация об авторах}

Нина Николаевна Ивашиненко, доктор экономических наук, профессор, руководитель Научно-образовательного центра исследований социальных систем, Нижегородский государственный университет им. Н.И. Лобачевского, пер. Университетский, 7-209, 603000 г. Нижний Новгород, Российская Федерация; почетный исследователь Школы социальных и политических наук, Университет Глазго, Юниверсити авеню, G12 8QQ г. Глазго, Великобритания, nni@fsn.unn.ru, nina.ivashinenko@glasgow.ac.uk, https://orcid.org/0000-0001-5242-169X

Михаил Леонидович Теодорович, доктор социологических наук, заведующий кафедрой социологии проектной деятельности и проконкурентного регулирования, Нижегородский государственный университет им. Н.И. Лобачевского, пер. Университетский, 7-209, 603000 г. Нижний Новгород, Российская Федерация, mt231@fsn.unn.ru, https://orcid.org/0000-0002-2136-9101

Алла Александровна Варызгина, кандидат социологических наук, старший преподаватель кафедры социологии проектной деятельности и проконкурентного регулирования, Нижегородский государственный университет им. Н.И. Лобачевского, пер. Университетский, 7-209, 603000 г. Нижний Новгород, Российская Федерация, alvar@fsn.unn.ru, https://orcid.org/0000-0002-1406-836X 